General and Supportive Care

\title{
Diabetes and pancreatic neuroendocrine tumours: Which interplays, if any?
}

\author{
Marco Gallo $^{\mathrm{a}, *}$, Rosaria Maddalena Ruggeri ${ }^{\mathrm{b}}$, Giovanna Muscogiuri ${ }^{\mathrm{c}}$, Genoveffa Pizza ${ }^{\mathrm{d}}$, \\ Antongiulio Faggiano $^{\mathrm{e}}$, Annamaria Colao ${ }^{\mathrm{e}}$, on behalf of NIKE Group \\ a Oncological Endocrinology Unit, Department of Medical Sciences, University of Turin, AOU Città della Salute e della Scienza di Torino, Turin, Italy \\ ${ }^{\mathrm{b}}$ Department of Clinical and Experimental Medicine, Unit of Endocrinology, University of Messina, Italy \\ ${ }^{\mathrm{c}}$ Ios and Coleman Medicina Futura Medical Centre, Naples, Italy \\ ${ }^{\mathrm{d}}$ Unit of Internal Medicine, Landolfi Hospital, Solofra, Avellino, Italy \\ e Department of Clinical Medicine and Surgery, University "Federico II", Naples, Italy
}

\section{A R T I C L E I N F O}

\section{Keywords:}

Neuroendocrine tumours

Diabetes

Nutrition

Lifestyle

Therapy

\begin{abstract}
A B S T R A C T
Pancreatic neuroendocrine tumours (PanNETs) represent an uncommon type of pancreatic neoplasm, whose incidence is increasing worldwide. As per exocrine pancreatic cancer, a relationship seems to exist between PanNETs and glycaemic alterations. Diabetes mellitus (DM) or impaired glucose tolerance often occurs in PanNET patients as a consequence of hormonal hypersecretion by the tumour, specifically affecting glucose metabolism, or due to tumour mass effects. On the other hand, pre-existing DM may represent a risk factor for developing PanNETs and is likely to worsen the prognosis of such patients. Moreover, the surgical and/or pharmacological treatment of the tumour itself may impair glucose tolerance, as well as antidiabetic therapies may impact tumour behaviour and patients outcome. Differently from exocrine pancreatic tumours, few data are available for PanNETs as yet on this issue. In the present review, the bidirectional association between glycaemic disorders and PanNETs has been extensively examined, since the co-existence of both diseases in the same individual represents a further challenge for the clinical management of PanNETs.
\end{abstract}

\section{Introduction}

Neuroendocrine tumours (NETs) constitute a heterogeneous group of neoplasms that can be associated with a broad range of local and systemic symptoms, related to tumour mass effects and/or to the secretion of several hormones, peptides, and biogenic amines [59,38]. They can arise almost anywhere in the body, even if the majority of NETs originate in the gastroenteropancreatic (GEP) tract or in the bronchopulmonary system (about $70 \%$ and $25 \%$ of NETs, respectively) [19]. The majority of NETs are sporadic, whereas $5-30 \%$ of cases can arise in complex hereditary endocrine disorders [23,24]. They can appear at all ages, but the highest incidence of sporadic GEP NETs is from the fifth decade onward [63].

Pancreatic NETs (PanNETs), accounting for 4-7\% of GEP NETs [28], are sometimes associated with impaired glucose metabolism and/or to overt diabetes mellitus, which may result from a direct tumour effect on the pancreas, from the release of substances interfering with insulin secretion and/or activity, or from the surgical and/or medical treatment of the tumour itself. Furthermore, the increased life expectancy of patients with NETs has increased the number of subjects developing health conditions typical of the elderly, such as diabetes and its complications [35].

Beyond sharing some specific features (average age of onset, ongoing increase of incidence and prevalence, and probably some risk factors), the co-existence of a PanNET and diabetes mellitus (DM) in the same patient poses some complex clinical questions, such as: the metabolic effects of NETs therapies; the potential effects of some antidiabetic therapies on the neuroendocrine system; which antidiabetic therapy to use; the prognostic effect of diabetes on the tumour, and so on. This manuscript aims to review published data on the risk factors for PanNETs, to describe the consequences of NETs therapies on glucose metabolism, and to address some of the more common challenges of DM treatment in patients with PanNETs.

\section{Epidemiology of PanNETs and diabetes}

Although initially considered rare tumours, the incidence of NETs has gradually increased from 1.9 to $6.9 / 100,000$ people per year during the last three decades, probably due to improvements in diagnostic techniques and increased attention from both clinicians and

\footnotetext{
* Corresponding author at: Via Genova 3, I-10126 Turin, Italy.

E-mail address: mgallo4@cittadellasalute.to.it (M. Gallo).

${ }^{1}$ Details of the NIKE Group is given in the Acknowledgement section.
} 
Table 1

Estimated incidence of pancreatic neuroendocrine tumours according to cancer registries. ${ }^{a}$

\begin{tabular}{ll}
\hline panNET & $\begin{array}{l}\text { Estimated incidence(per 1,000,000 people } \\
\text { per year) }\end{array}$ \\
\hline Non-functioning & $4-5$ \\
Insulinoma & $2-4$ \\
Gastrinoma & $0.5-4$ \\
Glucagonoma & 0.05 \\
VIPoma, somatostatinoma, others & $<0.025$ \\
\hline
\end{tabular}

a Oberg and Eriksson [62], Dasari et al. [19].

pathologists $[89,19]$. The crude incidence of sporadic NETs increases with age, and it peaks between 50 and 70 years, except for the carcinoid of the appendix, occurring earlier in the majority of cases. A slight overall higher incidence of NETs was observed for males (5.35) compared with females (4.76) [63]. Nowadays GEP NETs represent the second most common gastrointestinal neoplasm after colorectal adenocarcinoma [91]. It should also be noted that the prevalence of GEP NETs in autopsy series is significantly higher than that observed in clinical series.

In Western countries, the estimated clinical incidence of PanNETs is approximately 0.8/100,000 people [19], albeit PanNETs have been described in $0.5 \%-1.5 \%$ of autopsies. Altogether, PanNETs account for only $1-2 \%$ of pancreatic neoplasms $[62,52,19]$. However, a sharp increase in the incidence of nonfunctioning PanNETs was described over the past decades, greater than 3-fold increase [28,52,19]. Indeed most of PanNETs are nonfunctioning, with surgical and medical series suggesting insulinoma and gastrinoma as the most common functioning forms (Table 1). Due to the relative indolent clinical course of PanNETs (relative to that of exocrine pancreatic carcinoma), to improvements in treatment strategies, and to the long survival of many patients, PanNETs prevalence is also increasing having been estimated to account for approximately $10 \%$ of all pancreatic tumours despite the lower incidence $[28,91,19]$.

Conversely, diabetes mellitus (DM) is one of the main challenges of the 21st-century regarding global health. Despite relevant improvements in DM management, and declines in its rate of complications and mortality, the absolute numbers of people with DM is steadily increasing all over the world, with an unprecedented rise in low- and middle-income countries [61]. According to estimates from the International Diabetes Federation (IDF), there were 415 million people with DM in 2015, whereas the absolute number will reach 642 million by 2040 [44]. The IDF also estimates that by 2035 low- and middle-income countries will experience a $50 \%$ increase in the prevalence of impaired glucose tolerance (IGT, a form of prediabetes), compared with $41 \%$ increase in high income countries. It should be noted that, while the definition of DM and IGT is the same for the World Health Organization (WHO), the IDF, and the American Diabetes Association (ADA), impaired fasting glucose (IFG, the other condition of prediabetes) is defined as fasting plasma glucose level of $100-125 \mathrm{mg} / \mathrm{dL}(5.6-6.9 \mathrm{mmol} /$ mol) according to the ADA definition, whereas as $110-125 \mathrm{mg} / \mathrm{dL}$ (6.1-6.9 $\mathrm{mmol} / \mathrm{mol}$ ) according to the WHO/IDF definition. Therefore, the ADA IFG definition recognizes more subjects at increased risk of developing DM (and with significantly increased cardiovascular risk) compared with the WHO/IDF definition [27]. Furthermore, among people with DM impressive improvements in outcomes occurred in Western countries over the past two decades. As a consequence, the absolute number of people with DM is constantly increasing [61].

\section{Diabetes as a risk factor for PanNETs}

The pathogenesis and the risk factors for sporadic PanNETs are not well defined, since little epidemiologic research in this area has been performed. According to the small number of available studies, a family history of any cancer (and of PanNETs in particular), DM, and perhaps a high BMI, have all been suggested as risk factors for PanNETs $[40,13,97]$.

The association between DM and PanNETs has been examined by several case-control studies $[39,13,39,6]$ and by two meta-analyses $[41,53]$, which consistently indicated DM as a potential risk factor for the development of PanNETs. Leoncini and coll., in their recent systematic review and meta-analysis, reported a strong significant association between a history of DM and PanNETs, with a summary effect estimate of 2.76 (95\% CI 1.65-4.64) [53]. This effect was even higher when considering subjects with non-functioning PanNETs [6] and those with "recent onset DM" (ie, DM diagnosed in the 12 months before the diagnosis of a PanNET) (OR 12.80, 95\% CI 2.47-66.42). Together with gastric NETs, those of pancreatic origin are the GEP NETs where DM seems to represent the strongest risk factor.

It should be noted that obesity and DM have also been strongly associated with pancreatic ductal adenocarcinoma (PDAC), which is far more common than PanNETs, suggesting that NETs and adenocarcinomas might share some of the same risk factors, at least for this anatomical site $[41,53]$. Unfortunately, most of the studies considered in the meta-analysis did not provide data adjusted for important modifiable determinants of cancer risk such as diet, nutrition, physical activity, and the type of diabetes treatment.

The mechanisms linking DM to PanNETs development are largely unknown. The presence of the MEN-1 syndrome and the co-existence of a functioning PanNET may rise blood glucose levels, but it is a quite rare condition. Moreover, PanNETs may induce hyperglycaemia and peripheral insulin resistance by producing diabetogenic factors, similarly to advanced PDAC $[75,77]$. Therefore, DM can be an early paraneoplastic condition or a consequence of a tumour-induced impairment of glucose metabolism, instead of a real factor promoting cancer initiation ("reverse causality") $[16,64]$. Alternatively, DM may act as a mediator for chronic inflammation and intracellular oxidative stress, leading to DNA mutation and to the development of PanNETs [6]. In addition, the progressive tumoural disease may cause the obstruction of the pancreatic duct and the disruption of vasculature, promoting the destruction or atrophy of pancreatic tissue, ultimately worsening insulin secretion [77]. Existing evidence suggests that reverse causality is unlikely to be entirely responsible for the observed association between DM and PDAC, whereas little evidence is available for PanNETs [47]. Further research is necessary to clarify this issue.

\section{Mechanisms of diabetes onset in patients with PanNETs}

\section{Direct effect of functioning PanNETs}

In patients with PanNETs imbalance of glucose metabolism and DM may be due to an altered secretion of hormones that can induce hyperglycaemia and insulin resistance, such as with glucagonomas or somatostatinomas, or to decreased insulin secretion, as it is the case of patients who underwent pancreatic surgery [72].

\section{Glucagononoma}

Glucagonoma is an uncommon tumour arising from pancreatic neuroendocrine islet $\alpha$-cells secreting abundant glucagon. The estimated annual incidence of glucagonoma is $\sim 1$ case per $20,000,000$ individuals [17]. Mortality rates remain unclear, with fewer than 300 cases reported and the largest case series including only 21 patients. The typical clinical presentation of glucagonoma usually manifests when these tumours are over $4-5 \mathrm{~cm}$ in size and metastatic, particularly to the liver. Glucagon exerts its physiological role by increasing the hepatic glucose output and maintaining normal blood glucose level. Furthermore, glucagon exerts a catabolic role by reducing protein synthesis. Therefore, elevated glucagon levels result in amino acid catabolism and serum glucose elevation, which are considered to be responsible of the typical skin lesions and DM. 
The glucagonoma syndrome include a characteristic rash termed necrolytic migratory erythema ( $82 \%$ of patients), painful glossitis, cheilitis, angular stomatitis, normochromic normocytic anaemia (61\%), weight loss (90\%), mild DM (80\%), hypoaminoacidemia, low zinc levels, deep vein thrombosis (50\%), and depression (50\%). It goes by the acronym '4D syndrome', which stands for dermatosis, diabetes, deep vein thromboses, and depression [21].

Glucose tolerance in the glucagonoma syndrome may relate to tumour size. The fasting plasma glucagon level tends to be higher in patients with large hepatic metastases than in those without metastases. Massive hepatic metastases may decrease the ability of the liver to metabolize splanchnic glucagon, thus increasing peripheral plasma glucagon levels. Glucagon may not directly induce hyperglycaemia, unless metabolism of glucose by the liver is directly compromised. A variation in the molecular species of glucagon and its biologic potency can be present in each case $[57,78]$.

DM is a clinical hallmark of glucagonoma, although its severity is controversial. Only $40 \%$ of glucagonoma patients had overt DM at onset of symptoms, but about $90 \%$ went to develop it [17]. In the largest study group reported so far, although only 8 out of the 21 subjects with glucagonoma had overt DM at presentation, DM eventually developed in 16 patients, $75 \%$ of whom requiring insulin therapy. No patients had necrolytic migratory erythema clearly preceding DM [86].

\section{Somatostatinoma}

Somatostatinoma is quite rare, representing about $4 \%$ of GEP NETs [48], and its estimated incidence is about 1 in 40 million. Although this tumour secretes somatostatin, clinical presentation related to high somatostatin levels can be found in less than $10 \%$ of cases according to the tumour site, as well as intermittent somatostatin secretion [60]. Somatostatinoma syndrome typically includes DM, diarrhoea/steatorrhoea, gallbladder disease (particularly cholelithiasis), hypochlorhydria, and weight loss [66]. This syndrome is more common in pancreatic somatostatinomas than in the duodenal counterpart, owing to the higher levels of somatostatin secreted by pancreatic tumours.

DM has been reported in $75 \%$ of patients with pancreatic somatostatinomas, but only in $11 \%$ of cases with duodenal tumours. In all cases, DM was relatively mild and controlled with diet and oral antidiabetic agents, or with small doses of insulin. However, it is unclear whether the differential inhibition of insulin and diabetogenic hormones can explain the usual mild degree of hyperglycaemia and the rarity of ketoacidosis in patients with somatostatinoma. An "inhibitory syndrome" was reported in a malignant somatostatinoma, characterized by mild nonketonic hyperglycaemia, hyperchlorhydria, cholelithiasis, steatorrhoea, and weight loss [83]. In these tumours, the absence of ketoacidosis is thought to arise from the somatostatin-induced simultaneous suppression of the secretion of both insulin and glucagon.

Replacement of functional islet cell tissue by pancreatic tumour may be another reason for the development of DM in most patients with pancreatic somatostatinoma, contrasting with the low incidence in patients with somatostatinoma arising from the duodenum.

\section{Ectopic Cushing's syndrome due to a PanNET}

Cushing's syndrome due to ectopic production of CRH or ACTH is rare, accounting for less than $20 \%$ of all cases of ACTH-dependent Cushing. Even if bronchial carcinoids are the most frequent causes of ectopic Cushing's syndrome, PanNETs can also be responsible for CRH/ ACTH secretion. In a recently published multicenter retrospective analysis of 110 patients diagnosed with ectopic Cushing's syndrome, a PanNET was the source of ectopic secretion of CRH/ACTH in $15.5 \%$ of cases (mean age at diagnosis: 48.3 years), with DM as a clinical feature at diagnosis in over $60 \%$ of patients [20]. The majority of PanNETs secreting CRH/ACTH were well differentiated (G1 or G2), of big size ( $>4 \mathrm{~cm}$ diameter), with signs of local aggressiveness, and with distant metastases. Moreover, PanNETs showed a significantly worse outcome compared to bronchial carcinoids, and the presence of DM was an adverse prognostic factor.

Measurement of fasting glucose may underestimate the number of patients with Cushing's syndrome and DM, whereas glycated haemoglobin and post-prandial glucose levels are more helpful [35].

\section{Surgery (pancreatectomy)}

The resection of the distal part of the pancreas (tail or body) for neoplastic lesions may lead to insulin and glucagon deficiency, inducing diabetes (or worsening glycaemic control in patients with pre-existing DM), and making patients more vulnerable to severe hypoglycaemia. In a large study group, $4.8-39 \%$ of patients developed pancreatogenic diabetes postoperatively [81].

The diabetogenic effects of distant pancreatectomy are discussed in literature. Menge et al. evaluated the effects of a 50\% partial pancreatectomy on glucose homeostasis and insulin secretion in patients with pancreatitis, pancreatic carcinoma, and benign pancreatic tumours. The authors concluded that insulin secretion is reduced after pancreatic-head and -tail resection, but post-challenge glucose concentrations can paradoxically be improved after pancreatic head resection. Obesity and preoperative hyperglycaemia should be considered as risk factors for the development of hyperglycaemia and DM after pancreatic surgery [58].

In line with these data, a retrospective study evaluated the glucose profile of 82 patients after distant pancreatectomy. The authors demonstrated that blood glucose levels were higher in patients with established DM, while $35 \%$ of patients with pre-existing DM showed an improvement of glucose levels and a reduction of diabetic medications use. Insulin was the most common antidiabetic drug used for subjects showing worsened glucose levels ( $50 \%$ of patients), but $75 \%$ of patients treated with insulin had uncontrolled or undiagnosed diabetes preoperatively. The authors concluded that the worsening of glucose control after surgery may be due to preoperatively uncontrolled or undiagnosed DM, not only to beta cells reduction. On the other hand, the improvement of glycaemic control after pancreatic cancer resection may be due to insulin resistance caused by the tumour itself [55].

Another speculative hypothesis can be considered for the better glycaemic control often observed in patients with DM, after partial pancreatectomy. In fact, a glucose tolerance improvement could also be due to the weight loss induced by the failure of the exocrine function of the pancreatic tissue leftover, which could enhance peripheral insulin sensitivity. However, there are contradictory reports on this issue. Recently, Park et al. retrospectively reviewed 45 patients who had undergone radical resection for pancreatic adenocarcinoma: 34 patients underwent pancreaticoduodenectomy, 10 patients distal pancreatectomy, and one received total pancreatectomy. Even if no data about weight loss and malabsorption were reported in their analysis, they found that DM often improved after surgery: diabetes resolution occurred in $20-57 \%$ of patients after pancreaticoduodenectomy and in $13 \%$ of patients after distal pancreatectomy [65].

Indeed, albeit possible, only a large pancreatectomy can determine a malabsorption due to the failure of pancreatic exocrine function, which is generally well controlled by the rapid introduction of replacement therapy with pancreatic enzymes.

\section{Drug therapy}

Several drugs currently used for the medical management of NETs may induce hyperglycaemia through a variety of mechanisms, including alterations in insulin secretion and sensitivity, direct cytotoxic effects on pancreatic cells, and increases in glucose production or decrease in cell consumption $[25,18]$.

Somatostatin analogues (SSAs), which are commonly used as antisecretive and anti-proliferative agents in patients with NETs, may cause aberrations in glucose tolerance by their inhibitory effects on pancreatic endocrine secretion. The occurrence of hyperglycaemia after SSAs, and 
Table 2

Hyperglycaemia (all grades and Grade 3-4) in the main prospective clinical trials performed with everolimus in patients with neuroendocrine tumors.

\begin{tabular}{|c|c|c|c|c|c|}
\hline \multirow[t]{2}{*}{ Trial } & \multirow[t]{2}{*}{ Therapy } & \multirow[t]{2}{*}{ Patients (n) } & \multicolumn{2}{|c|}{ Hyperglycaemia (\%) } & \multirow[t]{2}{*}{ Primary NET site } \\
\hline & & & Any grade & Grade 3 or 4 & \\
\hline RADIANT-1 [92] & Everolimus \pm octreotide LAR & 160 & 13 & 4 & Pancreas \\
\hline RADIANT-2 [67] & Everolimus + octreotide LAR & 211 & 12 & 5 & Lung, ileum, pancreas, liver, colon, other \\
\hline RADIANT-3 [93] & Everolimus $v s$ placebo & 207 & 13 & 5 & Pancreas \\
\hline RADIANT-4 [90] & Everolimus $v s$ placebo & 205 & 10 & 3 & Lung, ileum, pancreas, colon \\
\hline COOPERATE-2 [51] & Everolimus \pm pasireotide LAR & 160 & $76^{\mathrm{a}} ; 27^{\mathrm{b}}$ & $37^{\mathrm{a}} ; 11^{\mathrm{b}}$ & Pancreas \\
\hline LUNA [26] & Everolimus \pm pasireotide LAR & 83 & $83^{\mathrm{a}} ; 46^{\mathrm{b}}$ & $29^{\mathrm{a}} ; 17^{\mathrm{b}}$ & Lung, thymus \\
\hline [14] & Everolimus + pasireotide & 21 & n.r. & $38 \%$ & Lung, ileum, pancreas \\
\hline [3] & Everolimus \pm octreotide LAR & 50 & 18 & 0 & Lung, ileum, pancreas, gut, unknown \\
\hline [15] & Everolimus + octreotide \pm bevacizumab & 150 & $12-14 \%$ & n.r. & Pancreas \\
\hline
\end{tabular}

n.r.: not reported.

a Everolimus + pasireotide LAR.

b Everolimus alone.

in particular after pasireotide, is among the most common adverse events (AEs) reported in clinical trials, second in frequency only to the well-known gastro-intestinal disturbances (diarrhoea and steatorrhea, constipation, gas and dysmotility, and gallstones) caused by the inhibitory effect of SSAs on gastro-intestinal and pancreatic functions [18].

SSAs affect glucose homoeostasis because of the expression of SSA receptor subtypes on the membranes of pancreatic islet-cells. As demonstrated by in vitro experiments on cultured isolated pancreatic islets, somatostatin inhibits beta-cell insulin secretion through SSTR2 and SSTR5 receptor subtypes. Somatostatin also inhibits glucagon secretion by pancreatic alpha-cells by activating the SSTR2 receptor subtype $[96,80]$.

By acting predominantly on the SSTR2 subtype, first generation SSAs (octreotide, lanreotide) have less of a detrimental effect on insulin/glucagon balance than pasireotide, the newer pan-somatostatin analogue, which inhibits insulin secretion much more potently than glucagon due to its high affinity for SSTR5. As a consequence, despite SSAs show similar AEs, disturbances of carbohydrate metabolism are more common and more severe with pasireotide than with first-generation SSAs. Indeed, no glucose alterations were recorded among the 85 participants to the PROMID trial (octreotide LAR vs placebo) [76], whereas in the CLARINET trial (lanreotide $v s$ placebo) hyperglycaemia occurred in 5 out of the 101 patients under treatment (i.e. 5\%) compared to none of the 100 patients of the placebo group [12]. These alterations were generally mild and dose dependent, and tended to resolve within the first weeks of treatment [18]. In a recent Phase III trial comparing pasireotide LAR with octreotide LAR in patients with treatment-refractory carcinoid syndrome, pasireotide was associated with a significantly higher rate of hyperglycaemia (28.3\% vs 5.3\%). Also the rate of Grade 3-4 hyperglycaemia was higher with pasireotide LAR $(9.4 \%)$ than with octreotide LAR $(1.8 \%)$, and two patients receiving pasireotide LAR discontinued treatment because of Grade 4 drug-related hyperglycaemia, while no patient in the octreotide LAR arm discontinued the study because of drug-related glycaemic AEs [88].

However, based on clinical data obtained in healthy volunteers and in patients with Cushing disease, pasireotide seems to exert short- and medium term hyperglycaemic effects that are well manageable with antidiabetic therapy, when necessary [74].

Noteworthy, SSAs should be used with caution in patients with insulinomas because they can worsen hypoglycaemia by suppressing glucagon secretion $[46,85]$. In patients with malignant insulinomas, mTOR inhibitors (everolimus) can control insulin secretion and hypoglycaemia $[49,45,8,31]$.

Metabolic disturbances, mainly hyperglycaemia, have also emerged as a common $\mathrm{AE}$ of the mTOR inhibitor everolimus, that selectively inhibits mTORC1 downstream of the phosphatidylinositol 3-kinase (PI3K)/Akt pathway [31]. As demonstrated in experimental studies, everolimus impairs glucose metabolism both inhibiting insulin secretion [71] and increasing insulin resistance, and lowering glucose transport and intracellular utilization in peripheral tissues [95]. These mechanisms may account for everolimus-associated hyperglycaemia observed clinically, particularly in patients with pre-existing alterations of carbohydrate metabolism. In the RADIANT-3 trial (everolimus $10 \mathrm{mg}$ daily $v s$ placebo), for example, hyperglycaemia was seen in $13 \%$ of patients on everolimus (Grade 3-4, 5\%). The frequency of Grade 3-4 hyperglycaemia was higher in patients with DM or baseline hyperglycaemia (15\%) than in those without pre-existing DM (3\%) [93]. The association of everolimus with first-generation SSAs (RADIANT-2 trial, everolimus $10 \mathrm{mg}$ daily plus octreotide LAR $30 \mathrm{mg}$ every 28 days $v s$ placebo plus octreotide LAR) did not worsen the risk of hyperglycaemia, and the AE profile was similar to that of RADIANT-3 trial. Also in a Phase II trial comparing the association of everolimus with the vascular endothelial growth factor (VEGF)-inhibitor bevacizumab vs everolimus alone in 150 patients receiving concomitant octreotide at the standard dosage, hyperglycaemia occurred with similar frequency in the two treatment arms (14\% and $12 \%$, respectively) $[50,15]$. In contrast, the combination of everolimus with the new SSA analogue pasireotide (COOPERATE-2 trial, everolimus plus pasireotide $v s$ everolimus alone) was associated to significantly higher rates of hyperglycaemia (all grades, $76 \%$ vs 27\%; Grade 3-4, 37\% vs 11\%) and DM (26\% vs 7\%) [51].

Hyperglycaemia may also be considered as an "on-target off-tumour effect" of the drug, i.e. an exaggerated pharmacologic effect that occurs when the target that is being inhibited in the tumour is also being inhibited in the normal tissue. So, it could be speculated that the appearance of hyperglycaemia as a major on-target off-tumour effect may correspond to a good on-tumour activity of everolimus and might be proposed as a predictive markers of treatment response, as per the ontarget side effects of other drugs (i.e. bevacizumab-induced hypertension) [98]. Hyperglycaemia, as reported as an AE in the main prospective clinical trials performed with everolimus in patients with NETs, is summarized in Table 2.

Finally, NETs patients are at risk for the development of hyperglycaemia from second-line treatments, including interferon- $\alpha$ (IFN- $\alpha$ ) and certain chemotherapeutic agents [42]. It is well-know that IFN- $\alpha$ may induce autoimmune type $1 \mathrm{DM}$ and may result in permanent insulin therapy dependency [9]. Streptozocin, a systemic chemotherapy agent approved for PanNETs, is cytotoxic to pancreatic beta-cells [29].

Clinicians should be aware of such metabolic AEs when using SSAs, mostly pasireotide, and/or everolimus to treat NETs. A number of recommendations can be proposed, including testing for impaired glucose regulation before initiating therapy and monitoring glycaemic control as an integral part of the follow-up. It is advisable to monitor blood glucose and HbA1c levels in all patients receiving such therapies, and to pay even more attention to those with overt DM, IGT, or impaired 
fasting hyperglycaemia. Patients with pre-existing alterations of glucose homeostasis and those with well-known risk factors for DM (such as previous gestational diabetes, obesity, and metabolic syndrome) are more likely to develop severe hyperglycaemia on therapy. Appropriate dietary and lifestyle measures represent the first-choice treatment of such drug-induced metabolic AEs. Introducing or intensifying antidiabetic therapy should be considered whenever blood glucose is out of its target value.

\section{Effects of antidiabetic drugs on PanNETs}

Recently, some antidiabetic drugs have been reported as an attractive therapy for PanNETs if surgical treatment is not curative. Since metformin has recently emerged as a potentially active agent in cancer prevention and treatment $[7,56,69]$, an ongoing trial (a single arm, prospective, single centre phase II study) is investigating the antiproliferative effect of metformin in combination with everolimus and octreotide LAR in pancreatic well-differentiated neuroendocrine tumour patients [70]. The potential molecular rationale is that metformin inhibits mTOR/S6K, preventing (differently from everolimus) the hyperactivation of Akt on Ser473 and causing the consequent reactivation of mTOR by hyperactive Akt [82].

Dipeptidyl Peptidase-4 (DPP4) inhibitors showed interesting results in the treatment of insulinoma in an animal experimental setting. A very recent study has hypothesized that a DPP-4 inhibitor (sitagliptin) could have antinflammatory properties due to the expression of DPP-4 (CD 26) on the surface of insulinoma cells. In fact the treatment with sitagliptin of rat insulinoma cells resulted in the suppression of the nuclear factor Nf-kappaB activation and inflammatory citochines expression, suggesting that sitagliptin could exert a direct antinflammatory action on islet beta cells [43]. Despite these encouraging findings, the use of incretin therapy (both Glucagon-Like Peptide [GLP]-1 receptor agonists and DPP-4 inhibitors) in patients with DM and a PanNET raises controversy. Even if these antidiabetic drugs are able to attain good metabolic control with few side effects, they also have a potential regenerative influence on pancreatic cells. In fact the administration of incretin therapy in patients with DM was associated by both increased exocrine cell proliferation and dysplasia (alfa cell hyperplasia, glucagon expressing microadenomas and neuroendocrine tumours) [11]. Furthermore, liraglutide (a GLP-1 receptor agonist) has been found to promote medullary thyroid cancer (MTC) -another neuroendocrine tumour- in rodents, even if the relevance of this finding in humans has been questioned [30]. However, liraglutide is currently contraindicated in people with a personal or family history of MTC in the USA.

However, at least in the diagnostic field, the use of GLP-1 receptor agonists could represent a promising approach. GLP-1 receptors are expressed in over $90 \%$ of insulinomas, at a mean density twice that of SSTR2 [73]. Early preclinical studies demonstrated that ${ }^{123}$ I-GLP-1 and ${ }^{123}$ I-exendin 3 accumulate in rat insulinomas (RINm5F) subcutaneously implanted in NEDH rats and that these tumours can be detected scintigraphically [36]. Due to the washout of radionuclides after internalization, radioiodinated peptides have a relatively low uptake in vivo $[79,37]$. Further, a high uptake of DTPA conjugated [Lys40] exendin-4 labelled with ${ }^{111}$ In has been reported in the Rip1tag2 spontaneous insulinoma mouse model. The conjugation of ${ }^{111}$ In with [Lys 40 (DTPA)] exendin 4 has been reported to markedly inhibit the growth of insulinomas in Rp1Tag2 mice [87]. Based on these findings, the use of GLP-1 receptor agonists has been hypothesized to become a useful tool to increase the detection rate of insulinomas, which are often hardly to visualize. Also the conjugation of exendin 3 with DOTA and ${ }^{68} \mathrm{Ga}$ has been described to successfully detect small insulinomas, albeit already inducing symptomatic hypoglycaemia [10].

\section{Prognostic role of diabetes on PanNETs}

DM and glucose intolerance are commonly associated with increased long-term, all-cause mortality in oncologic patients [4]. Since NET patients are generally at risk of having concomitant DM or of experiencing impaired glucose metabolism from a variety of therapies, the potential impact of glycaemic alterations on disease progression and survival is a relevant issue. Unfortunately, few data are available for NETs as yet.

Up to date, most studies have been mainly focused on the association between DM and the risk of developing PanNETs, as a similar association is well established for exocrine pancreatic cancer $[5,54]$. Clinical studies [6,39,13,40] and meta-analyses [41,53] consistently indicated DM as a potential risk factor for PanNETs. However, only one of the above mentioned studies provided data on the influence of such risk factors, including DM, on tumour progression and patients outcome [13]. In this report, pre-existing diabetes was significantly associated with metastatic disease at the time of diagnosis, suggesting a more aggressive behaviour of the tumour in the presence of this comorbidity. These findings were not confirmed by Ben and co-workers, who failed to demonstrate any association between advanced disease staging and a history of DM in their patients with non-functioning PanNETs [6]. More recently, our study group reported preliminary data on the nutritional and metabolic features of a cohort of patients suffering from nonfunctioning NETs, demonstrating that the presence of metabolic disorders was associated with a more severe disease. In particular, a greater tumour size at diagnosis was reported in subjects with diabetes [32]. Also Pusceddu et al. provided data on the potential correlation among glucose metabolism, diabetes treatment, and disease prognosis in a small cohort of 31 patients with PanNETs receiving everolimus in association with octreotide LAR [69]. Quite surprisingly, patients with DM had a significantly longer progression-free survival (PFS) compared with the normoglycaemic group (29 vs 11 months; $\mathrm{P}=0.0018$ ). However, subdividing DM patients on the basis of their antidiabetic treatment, PFS in patients treated with metformin was twice as long as that of subjects on insulin ( 36 vs 17 months), suggesting that metformin rather than DM may be the protective variable [69]. A more recent study evaluated the risk of developing DM and its effects on all-cause mortality after radiopeptide therapy for NETs [84]. In this report neither radiopeptide therapy appeared to increase the risk of developing DM nor DM coexistence increased the mortality of NET patients undergoing receptor-targeted radiopeptide therapy [84]. Finally, in a multicentric Italian study evaluating the prognostic factors affecting survival in NETs patients with ectopic Cushing's syndrome, DM was among the negative predictive factors for survival, along with hypercortisolism, hypokalemia, and distant metastases [20]. However, findings obtained in this particular setting cannot be extended to all NETs patients. Further research focusing on the association between glycaemic control and adverse outcomes in NETs patients, who are at risk for treatment-induced hyperglycaemia, is required.

\section{Diabetes management in patients with PanNETs}

Diabetes and PanNETS share another aspect, which pertains their therapeutic approach: for both conditions first line therapy is generally well-established, whereas difficulties concern the optimal sequential algorithm for people not reaching treatment goals. When tolerated and not contraindicated, metformin is the preferred initial pharmacologic agent for the treatment of type 2 diabetes. After failure of metformin monotherapy many other antidiabetic drugs are available, nowadays, and the choice of pharmacologic agents should be individualized based both on patients and drugs features [22].

For their part, SSAs remain the recommended first-line treatment for most somatostatin-receptor-expressing G1/G2 PanNETs, whether functioning or not. Further treatment decision should be based on tumour characteristics, tumour related symptoms, comorbidities, and the 


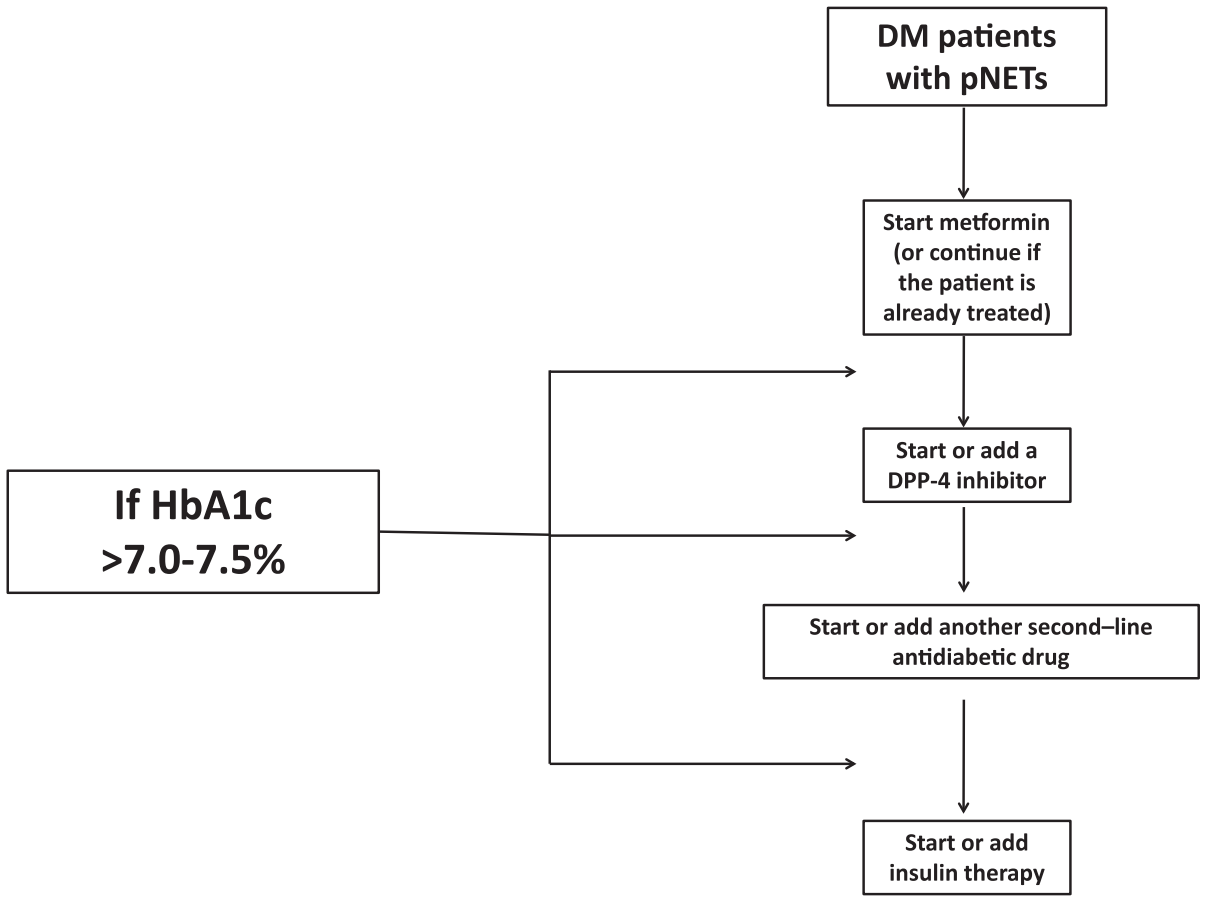

Fig. 1. Pharmacological treatment of diabetes in patients with PanNETs. When glucose control is not achieved with lifestyle and metformin, DPP-4 inhibitors represent a promising approach. After the failure of this combination, we suggest to add a second-line antidiabetic drug as suggested by current recommendations [2,34]. After the failure of oral antidiabetic drugs, insulin is required. DM = Diabetes Mellitus. local availability of various alternatives, but the ideal sequence of drugs after (or together with) SSAs is still a matter of debate [1].

The management of diabetes in patients with PanNETs should be generally based on the current recommendations for the treatment of type $2 \mathrm{DM}$, whether diabetes is already known or hyperglycaemia results as a consequence of pharmacological and/or surgical treatment $[2,34]$. Clearly, the aggressiveness of glucose control should be set taking into consideration numerous aspects (life expectancy, relevant comorbidities, resources and support system), and individualized on the basis of patients' characteristics. Thus, individually tailored glycaemic targets of HbA1c level $<7.0-7.5 \%$ are adequate, provided that the patient life-expectancy is long enough and that prevention of microvascular disease is an issue. Conversely, for patients with a poorer prognosis or in the cases where the treating clinician aims at minimizing the risk of hypoglycaemia, a less stringent glycaemic goal may better fit for the purpose (e.g., a HbA1c target of 7.6-8.5\%) [33].

Diabetes management should include dietary changes and physical activity. However, the therapeutic approach should take into account the underlying causes responsible for glucose imbalance (pre-existent T2DM, secondary DM due to pancreatic surgery, to functioning syndromes, or to the specific drugs employed to treat the tumour), the presence of nutritional problems, liver and renal function, and so on. However, data about diabetes therapy for patients with PanNETs are still lacking and further studies are strongly warranted.

When hyperglycaemia is mild, cancer patients can be treated with non-insulin antidiabetic drugs, preferentially addressing the pathophysiological mechanisms behind DM. This means that when DM is due to a glucagonoma, a somatostatinoma, or a PanNET known to produce ACTH with ectopic Cushing's syndrome, where the hypersecretion of counterregolatory hormones induces insulin resistance, metformin and glitazones may be preferred (even if pioglitazone may further increase the risk of bone loss and fracture due to hypercortisolism). Conversely, insulin therapy (rarely sulphonylureas and glinides) should be preferred for patients with impaired insulin secretion (DM secondary to pancreatic surgery or DM induced by SSAs). The recently introduced sodium-glucose co-transporter-2 (SGLT2) inhibitors could be employed both in patients with insulin resistance and in those with altered insulin secretion, provided that the renal function is preserved and the patient is not prone to diabetic ketoacidosis.
Nevertheless, as previously reported, treatment with metformin has been suggested to delay or slow the progression of different tumours, including PanNETs, with a potential favourable influence on patients survival. Therefore, the use of metformin should be contemplated as a first choice also in the oncological setting $[94,68,69]$. Further, metformin inhibits the hyperactivation of Akt on Ser473 that is secondary to everolimus therapy, thus potentiating its efficacy [82]. Potential gastrointestinal side effects of metformin should be taken into account, especially in patients already suffering from gastrointestinal discomfort as a result of the PanNET (e.g., VIPoma or gastrinoma related diarrhoea) or of the drugs employed for its treatment (SSAs).

As a second choice, DPP-4 inhibitors represent a promising approach being a class of drugs with few side effects. Furthermore, an antinflammatory effect on PanNETs has recently been reported [43]. Regarding GLP-1 receptor agonists, their potential proliferative properties make them an unsuitable class of drugs for the treatment of PanNET-related DM [11]. Finally, insulin therapy is usually required for patients on active anticancer therapy as well as for diabetic patients already on insulin therapy (Fig. 1).

\section{Conclusions}

Unexpectedly, PanNETs and DM share many aspects: both of them are conditions whose incidence and prevalence are increasing, they are often indolent in their natural history causing long term relevant consequences, and they both can exert unfavourable effects on each other prognosis. More and more therapies are nowadays available for their management, but the most effective sequence for each patient is a matter of debate requiring an individualized approach. Furthermore, along with obesity, DM is one of the few risk factors already known for PanNETS, and some of the drugs employed to treat both DM and PanNETs can exert some effects on the other one. Lastly, the co-existence of both the conditions in the same subject imposes additional challenges to the treating physicians. Hopefully, future research and clinical studies will further provide endocrinologists valuable insights, empirical answers, and additional evidence to deal appropriately with these challenges. 


\section{Compliance with Ethical standards}

\section{Funding: None.}

Conflict of Interest: The authors declare that there is no conflict of interest that could be perceived as prejudicing the impartiality of the research reported.

Ethical approval: This article does not contain any studies with human participants or animals performed by any of the authors.

\section{Acknowledgements}

This review is part of the 'NIKE' project (Neuroendocrine tumours Innovation Knowledge and Education) led by Prof Annamaria Colao, which aims at increasing the knowledge on NETs.

We would like to acknowledge all the Collaborators of this project: Manuela Albertelli, Sergio Baldari, Roberto Baldelli, Antonio Bianchi, Gerardo Botti, Arturo Brunetti, Luigi Buonaguro, Roberto Buzzoni, Luigi Camera, Davide Campana, Carlo Carnaghi, Luisa Circelli, Vittorio Colantuoni, Jorgelina Coppa, Alberto Cuocolo, Carlo Curcio, Maria Vittoria Davi', Federica De Cicco, Sabino De Placido, Gaetano De Rosa, Nicolina De Rosa, Marialaura Del Basso De Caro, G. Delle Fave, Carla Di Dato, Sergio Di Molfetta, Alessandra Dicitore, Massimo Falconi, Giuseppe Fanciulli, Piero Ferolla, Diego Ferone, Francesco Ferraù, Angelina Filice, Francesco Fiore, Pietro Forestieri, Elisa Giannetta, Dario Giuffrida, Federica Grillo, Erika Maria Grossrubatscher, Elia Guadagno, Valentina Guarnotta, Leo J Hofland, Andrea Isidori, Elda Kara, Stefano La Rosa, Andrea Lenzi, Fabio Lo Calzo, Francesco Logoluso, Gabriele Luppi, Pasqualino Malandrino, Marco Manzoni, Chiara Martini, Sara Massironi, Erika Messina, Roberta Modica, Vincenzo Montesarchio, Vincenzo Napolitano, Oscar Nappi, Kjell Öberg, Francesco Panzuto, Mauro Papotti, Anna Pia, Carmine Pinto, Alessandro Piovesan, Sara Pusceddu, Paola Razzore, Francesca Rota, Manila Rubino, Concetta Sciammarella, Francesca Spada, Annibale Versari, Giovanni Vitale, Maria Chiara Zatelli, and Alessandro Zerbi.

\section{References}

[1] Alonso-Gordoa T, Díez JJ, Molina J, Reguera P, Martínez-Sáez O, Grande E. An overview on the sequential treatment of pancreatic neuroendocrine tumors (pNETs). Rare Cancers Therapy 2015;3:13-33.

[2] American Diabetes Association. Standards of medical care in diabetes. Diabetes Care 2017;40(S1):S1-135.

[3] Bajetta E, Catena L, Fazio N, Pusceddu S, Biondani P, Blanco G, et al. Everolimus in combination with octreotide long-acting repeatable in a first-line setting for patients with neuroendocrine tumors: an ITMO group study. Cancer 2014;120(16):2457-63. http://dx.doi.org/10.1002/cncr.28726.

[4] Barone BB, Yeh HC, Snyder CF, Peairs KS, Stein KB, Derr RL, et al. Long-term allcause mortality in cancer patients with preexisting diabetes mellitus: a systematic review and meta-analysis. JAMA 2008;300:2754-64.

[5] Ben Q, Xu M, Ning X, Liu J, Hong S, Huang W, et al. Diabetes mellitus and risk of pancreatic cancer: A meta-analysis of cohort studies. Eur J Cancer 2011:47:1928-37. http://dx.doi.org/10.1016/j.ejca.2011.03.003.

[6] Ben Q, Zhong J, Fei J, Chen H, Yv L, Tan L, et al. Risk Factors for sporadic pancreatic neuroendocrine tumors: a case-control study. Sci Rep 2016;6:36073. http://dx.doi. org $/ 10.1038 /$ srep36073.

[7] Ben Sahra I, Le Marchand-Brustel Y, Tanti JF, Bost F. Metformin in cancer therapy: a new perspective for an old antidiabetic drug? Mol Cancer Ther 2010;9:1092-9.

[8] Bernard V, Lombard-Bohas C, Taquet MC, Caroli-Bosc FX, Ruszniewski P, Niccoli P, et al. Efficacy of everolimus in patients with metastatic insulinoma and refractory hypoglycemia. Eur J Endocrinol 2013;168:665-74.

[9] Bosi E, Minelli R, Bazzigaluppi E, Salvi M. Fulminant autoimmune Type 1 diabetes during interferon-alpha therapy: a case of Th1-mediated disease? Diabet Med 2001;18:329-32.

[10] Brom M, Oyen WJ, Joosten L, Gotthardt M, Boerman OC. 68Ga-labelled exendin-3, a new agent for the detection of insulinomas with PET. Eur J Nucl Med Mol Imaging 2010;37:1345-55. http://dx.doi.org/10.1007/s00259-009-1363-y.

[11] Butler AE, Campbell-Thompson M, Gurlo T, Dawson DW, Atkinson M, Butler PC. Marked expansion of exocrine and endocrine pancreas with incretin therapy in humans with increased exocrine pancreas dysplasia and the potential for glucagonproducing neuroendocrine tumors. Diabetes 2013;62:2595-604.

[12] Caplin ME, Pavel M, Ćwikła JB, Phan AT, Raderer M, Sedláčková E, et al. Lanreotide in metastatic enteropancreatic neuroendocrine tumors. N Engl J Med 2014:371:224-33.

[13] Capurso G, Falconi M, Panzuto F, Rinzivillo M, Boninsegna L, Bettini R, et al. Risk factors for sporadic pancreatic endocrine tumors: a case-control study of prospectively evaluated patients. Am J Gastroenterol 2009;104:3034-41. http://dx. doi.org/10.1038/ajg.2009.466.

[14] Chan JA, Ryan DP, Zhu AX, Abrams TA, Wolpin BM, Malinowski P, et al. Phase I study of pasireotide (SOM 230) and everolimus (RAD001) in advanced neuroendocrine tumors. Endocr Relat Cancer 2012;19:615-23. http://dx.doi.org/10. 1530/ERC-11-0382.

[15] Chan DL, Segelov E, Singh S. Everolimus in the management of metastatic neuroendocrine tumours. Therapeutic Adv Gastroenterol 2017;10:132-41. http://dx doi.org/10.1177/1756283X16674660.

[16] Chari ST, Leibson CL, Rabe KG, Timmons LJ, Ransom J, de Andrade M, et al. Pancreatic cancer-associated diabetes mellitus: prevalence and temporal association with diagnosis of cancer. Gastroenterology 2008;134:95-101.

[17] Chastain MA. The glucagonoma syndrome: a review of its features and discussion of new perspectives. Am J Med Sci 2001;321:306-20.

[18] Chung C. Management of neuroendocrine tumors. Am J Health-Syst Pharmacy 2016;73:1729-44.

[19] Dasari A, Shen C, Halperin D, Zhao B, Zhou S, Xu Y, et al. Trends in the incidence, prevalence, and survival outcomes in patients with neuroendocrine tumors in the United States. J Am Med Assoc Oncol 2017. http://dx.doi.org/10.1001/jamaoncol. 2017.0589.

[20] Davì MV, Cosaro E, Piacentini S, Reimondo G, Albiger N, Arnaldi G, et al. Prognostic factors in ectopic Cushing's syndrome due to neuroendocrine tumors: a multicenter study. Eur J Endocrinol 2017;176:451-9. http://dx.doi.org/10.1530/ EJE-16-0809.

[21] Eldor R, Glaser B, Fraenkel M, Doviner V, Salmon A, Gross DJ. Glucagonoma and the glucagonoma syndrome - cumulative experience with an elusive endocrine tumour. Clin Endocrinol (Oxford) 2011;74:593-8.

[22] Esposito K, Gentile S, Candido R, De Micheli A, Gallo M, Medea G, et al. Management of hyperglycemia in type 2 diabetes: evidence and uncertainty. Cardiovascular Diabetol 2013;12:81. http://dx.doi.org/10.1186/1475-2840-12-81.

[23] Faggiano A, Ramundo V, Circelli L, Colao A. Hereditary neuroendocrine tumor syndromes. In: Fedele Monica, editor. Hot Topics in endocrine and endocrine-related diseases, InTech; 2013. p 1-26 ISBN 978-953-51-1080-4. http://dx.doi.org/ 10.5772/53841.

[24] Faggiano A, Ferolla P, Grimaldi F, Campana D, Manzoni M, Davì MV, et al. Natural history of gastro-entero-pancreatic and thoracic neuroendocrine tumors. Data from a large prospective and retrospective Italian epidemiological study: the NET management study. J Endocrinol Invest 2012;35:817-23. http://dx.doi.org/10.3275/ 8102.

[25] Fathallah N, Slim R, Larif S, Hmouda H, Ben Salem C. Drug-induced hyperglycaemia and diabetes. Drug Saf 2015;38:1153-68. http://dx.doi.org/10.1007/ s40264-015-0339-z.

[26] Ferolla P, Brizzi MP, Meyer T, Mansoor W, Mazieres J, Do Cao C, et al. Efficacy and safety of long-acting pasireotide or everolimus alone or in combination in patients with advanced carcinoids of the lung and thymus (LUNA): an open-label, multicentre, randomised, phase 2 trial. Lancet Oncol 2017 Dec;18(12):1652-64. http:// dx.doi.org/10.1016/S1470-2045(17)30681-2.

[27] Filippatos TD, Rizos EC, Gazi IF, Lagos K, Agouridis D, Mikhailidis DP, et al. Differences in metabolic parameters and cardiovascular risk between American Diabetes Association and World Health Organization definition of impaired fasting glucose in European Caucasian subjects: a cross-sectional study. Arch Med Sci 2013;9:788-95. http://dx.doi.org/10.5114/aoms.2013.38671.

[28] Fitzgerald TL, Hickner ZJ, Schmitz M, Kort EJ. Changing incidence of pancreatic neoplasms: a 16-year review of statewide tumor registry. Pancreas 2008;37:134-8. http://dx.doi.org/10.1097/MPA.0b013e318163a329.

[29] Furman BL. Streptozotocin-induced diabetic models in mice and rats. Curr Protocols Pharmacol 2015;70:5.47.1-20. http://dx.doi.org/10.1002/0471141755. ph0547s70.

[30] Gallo M. Thyroid safety in patients treated with liraglutide. J Endocrinol Invest 2013;36:140-5.

[31] Gallo M, Malandrino P, Fanciulli G, Rota F, Faggiano A, Colao A. NIKE Group 2017a Everolimus as first line therapy for pancreatic neuroendocrine tumours: current knowledge and future perspectives. J Cancer Res Clin Oncol 2017;143:1209-1224. http://dx.doi.org/10.1007/s00432-017-2407-5.

[32] Gallo M, Muscogiuri G, Pizza G, Ruggeri MR, Barrea L, Faggiano A, et al. on behalf of NIKE Group 2017b The management of neuroendocrine tumors: a nutritional viewpoint. Crit Rev Food Sci Nutrition 2017,Oct 11:0. http://dx.doi.org/10.1080/ 10408398.2017.1390729.

[33] Gallo M, Muscogiuri G, Felicetti F, Faggiano A, Trimarchi F, Arvat E, et al. Adverse glycaemic effects of cancer therapy: indications for a rational approach to cancer patients with diabetes. Metabolism 2017c, Oct 6. pii: S0026-0495(17)30265-2. http://dx.doi.org/10.1016/j.metabol.2017.09.013.

[34] Garber AJ, Abrahamson MJ, Barzilay JI, Blonde L, Bloomgarden ZT, Bush MA, et al. Consensus statement by the American Association Of Clinical Endocrinologists and American College of Endocrinology on the comprehensive type 2 diabetes management algorithm - 2017 Executive Summary. Endocrine Practice 2017;23:207-38. http://dx.doi.org/10.4158/EP161682.CS.

[35] Ghigo E, Porta M. Diabetes secondary to endocrine and pancreatic disorders. Front Diabetes 2014;22:64-76. Basel, Karger. http://dx.doi.org/10.1159/000357248.

[36] Gotthardt M, Fischer M, Naeher I, Holz JB, Jungclas H, Fritsch HW, et al. Use of the incretin hormone glucagon-like peptide-1 (GLP-1) for the detection of insulinomas: initial experimental results. Eur J Nucl Med Mol Imaging 2002;29:597-606.

[37] Hassan M, Eskilsson A, Nilsson C, Jonsson C, Jacobsson H, Refai E, et al. In vivo dynamic distribution of 131I-glucagon-like peptide-1 (7-36) amide in the rat studied by gamma camera. Nucl Med Biol 1999;26:413-20. 
[38] Gustafsson BI, Kidd M, Modlin IM. Neuroendocrine tumors of the diffuse neuroendocrine system. Curr Opin Oncol 2008;20:1-12.

[39] Halfdanarson TR, Bamlet WR, McWilliams RR, Hobday TJ, Burch PA, Rabe KG, et al. Risk factors for pancreatic neuroendocrine tumors (PNETs): a clinic-based case-control study. Pancreas 2014;43:1219-22. http://dx.doi.org/10.1097/MPA. 0000000000000234

[40] Hassan MM, Phan A, Li D, Dagohoy CG, Leary C, Yao JC. Risk factors associated with neuroendocrine tumors: a U.S.-based case-control study. Int J Cancer 2008;123:867-73. http://dx.doi.org/10.1002/ijc.23529.

[41] Haugvik SP, Hedenström P, Korsæth E, Valente R, Hayes A, Siuka D, et al. Diabetes, smoking, alcohol use, and family history of cancer as risk factors for pancreatic neuroendocrine tumors: a systematic review and meta-analysis.

Neuroendocrinology 2015;101:133-42. http://dx.doi.org/10.1159/000375164.

[42] Hershey DS, Bryant AL, Olausson J, Davis ED, Brady VJ, Hammer M. Hyperglycemic-inducing neoadjuvant agents used in treatment of solid tumors: a review of the literature. Oncol Nurs Forum 2014;41:E343-54. http://dx.doi.org/10 1188/14.ONF.E343-E354.

[43] Hu X, Liu S, Liu X, Zhang J, Liang Y, Li Y. DPP-4 (CD26) inhibitor sitagliptin exerts anti-inflammatory effects on rat insulinoma (RINm) cells via suppressing NF-кB activation. Endocrine. 2017;55(3):754-63.

[44] IDF Diabetes Atlas. 7th ed. Brussels, Belgium: International Diabetes Federation; 2015.

[45] Jensen RT. Endocrine neoplasms of the pancreas. In: Yamada T, Alpers DH, Kaplowitz N, editors. Textbook of gastroenterology. 5th ed.Oxford: Blackwel International Diabetes Federation; 2008.

[46] Jensen RT, Cadiot G, Brandi ML, de Herder WW, Kaltsas G, Komminoth P, et al. ENETS Consensus Guidelines for the management of patients with digestive neuroendocrine neoplasms: functional pancreatic endocrine tumor syndromes. Neuroendocrinology 2012;95:98-119. http://dx.doi.org/10.1159/000335591.

[47] Johnson JA, Carstensen B, Witte D, Bowker SL, Lipscombe L, Renehan AG. Diabetes and Cancer Research Consortium 2012 Diabetes and cancer (1): evaluating the temporal relationship between type 2 diabetes and cancer incidence. Diabetologia 2012;55:1607-18. http://dx.doi.org/10.1007/s00125-012-2525-1.

[48] Klöppel G, Anlauf M. Epidemiology, tumour biology and histopathological classification of neuroendocrine tumours of the gastrointestinal tract. Best Pract Res Clin Gastroenterol 2005;19:507-17.

[49] Kulke MH, Bergsland EK, Yao JC. Glycemic control in patients with insulinoma treated with everolimus. N Engl J Med 2009;360:195-7.

[50] Kulke M, Niedzwiecki D, Foster N, Fruth B, Kunz P, Kennecke, H, et al. Randomized phase II study of everolimus (E) versus everolimus plus bevacizumab $(E+B)$ in patients (Pts) with locally advanced or metastatic pancreatic neuroendocrine tumors (pNET), CALGB 80701 (Alliance). J Clin Oncol 2015;33(suppl.): abstract 4005

[51] Kulke MH, Ruszniewski P, Van Cutsem E, Lombard-Bohas C, Valle JW, De Herder WW, et al. A randomized, open-label, phase 2 study of everolimus in combination with pasireotide LAR or everolimus alone in advanced, well-differentiated, progressive pancreatic neuroendocrine tumors: COOPERATE-2 trial. Ann Oncol 2017;28:1309-15. http://dx.doi.org/10.1093/annonc/mdx078.

[52] Lawrence B, Gustafsson BI, Chan A, Svejda B, Kidd M, Modlin IM. The epidemiology of gastroenteropancreatic neuroendocrine tumors. Endocrinol Metabolism Clin North Am 2011;40:1-18, vii. http://dx.doi.org/10.1016/j.ecl.2010.12.005.

[53] Leoncini E, Carioli G, La Vecchia C, Boccia S, Rindi G. Risk factors for neuroendocrine neoplasms: a systematic review and meta-analysis. Ann Oncol 2016;27:68-81. http://dx.doi.org/10.1093/annonc/mdv505.

[54] Liao WC, Tu YK, Wu MS, Lin JT, Wang HP, Chien KL. Blood glucose concentration and risk of pancreatic cancer: systematic review and dose-response meta-analysis. Br Med J 2015;349:g7371.

[55] Liu A, Carmichael KA, Schallom ME, Klinkenberg WD. Retrospective review of postoperative glycemic control in patients after distal pancreatectomy. Int J Surgery 2017;41:86-90.

[56] Liu B, Fan Z, Edgerton SM, Deng XS, Alimova IN, Lind SE, et al. Metformin induces unique biological and molecular responses in triple negative breast cancer cells. Cell Cycle 2009;8:2031-40.

[57] Lobo I, Carvalho A, Amaral C, Machado S, Carvalho R. Glucagonoma syndrome and necrolytic migratory erythema. Int J Dermatol 2010;49:24-9.

[58] Menge BA, Schrader H, Breuer TG, Dabrowski Y, Uhl W, Schmidt WE, et al. Metabolic consequences of a $50 \%$ partial pancreatectomy in humans. Diabetologia 2009;52:306-17.

[59] Modlin IM, Oberg K, Chung DC, Jensen RT, de Herder WW, Thakker RV, et al. Gastroenteropancreatic neuroendocrine tumours. Lancet Oncol 2008;9:61-72.

[60] Mozell E, Stenzel P, Woltering EA, Rösch J, O'Dorisio TM. Functional endocrine tumors of the pancreas: clinical presentation, diagnosis, and treatment. Curr Probl Surg 1990;27:301-86.

[61] Narayan KM. Type 2 Diabetes: why we are winning the battle but losing the war? 2015 Kelly west award lecture. Diabetes Care 2016;39:653-63. http://dx.doi.org/ 10.2337/dc16-0205.

[62] Öberg K, Eriksson B. Endocrine tumours of the pancreas. Best Pract Res Clin Gastroenterol 2005;19:753-81.

[63] Öberg K, Knigge U, Kwekkeboom D, Perren A. Group EGW. Neuroendocrine gastroentero-pancreatic tumors: ESMO Clinical Practice Guidelines for diagnosis, treatment and follow-up. Ann Oncol/ESMO 2012;23(Suppl 7):vii 124-130. http://dx. doi.org/10.1093/annonc/mds295.

[64] Pannala R, Leirness JB, Bamlet WR, Basu A, Petersen GM, Chari ST. Prevalence and clinical profile of pancreatic cancer-associated diabetes mellitus. Gastroenterology 2008;134:981-7. http://dx.doi.org/10.1053/j.gastro.2008.01.039.

[65] Park SY, Park KM, Shin WY, Choe YM, Hur YS, Lee KY, et al. Functional and morphological evolution of remnant pancreas after resection for pancreatic adenocarcinoma. Medicine (Baltimore) 2017;96:e7495. http://dx.doi.org/10.1097/ MD.0000000000007495.

[66] Patel YC, Ruggere D, Malaisse-Lagae F, Orci L. Alterations in somatostatin and other islet cell functions in the spontaneously diabetic BB Wistar rat: biochemical and morphological characterization. Metabolism 1983:32:18-25.

[67] Pavel ME, Hainsworth JD, Baudin E, Peeters M, Hörsch D, Winkler RE, et al. RADIANT-2 Study Group. Everolimus plus octreotide long-acting repeatable for the treatment of advanced neuroendocrine tumours associated with carcinoid syndrome (RADIANT-2): a randomised, placebo-controlled, phase 3 study. Lancet 2011 Dec 10;378(9808):2005-12. http://dx.doi.org/10.1016/S0140-6736(11)61742-X.

[68] Pierotti MA, Berrino F, Gariboldi M, Melani C, Mogavero A, Negri T, et al. Targeting metabolism for cancer treatment and prevention: metformin, an old drug with multi-faceted effects. Oncogene 2013;32:1475-87.

[69] Pusceddu S, Buzzoni R, Vernieri C, Concas L, Marceglia S, Giacomelli L, et al. Metformin with everolimus and octreotide in pancreatic neuroendocrine tumor patients with diabetes. Future Oncol 2016;12:1251-60. http://dx.doi.org/10.2217/ fon-2015-0077.

[70] Pusceddu S, de Braud F, Concas L, Bregant C, Leuzzi L, Formisano B, et al. Rationale and protocol of the MetNET-1 trial, a prospective, single center, phase II study to evaluate the activity and safety of everolimus in combination with octreotide LAR and metformin in patients with advanced pancreatic neuroendocrine tumors. Tumori 2014;100:e286-9. http://dx.doi.org/10.1700/1778.19298.

[71] Rachdi L, Balcazar N, Osorio-Duque F, Elghazi L, Weiss A, Gould A, et al. Disruption of Tsc2 in pancreatic beta cells induces beta cell mass expansion and improved glucose tolerance in a TORC1-dependent manner. Proc Nat Acad Sci USA 2008:105:9250-5.

[72] Resmini E, Minuto F, Colao A, Ferone D. Secondary diabetes associated with principal endocrinopathies: the impact of new treatment modalities. Acta Diabetol 2009;46:85-95.

[73] Reubi JC, Waser B. Concomitant expression of several peptide receptors in neuroendocrine tumours: molecular basis for in vivo multireceptor tumour targeting. Eur J Nucl Med Mol Imaging 2003;30:781-93.

[74] Reznik Y, Bertherat J, Borson-Chazot F, Brue T, Chanson P, Cortet-Rudelli C, et al Management of hyperglycaemia in Cushing's disease: experts' proposals on the use of pasireotide. Diabetes Metabolism 2013;39:34-41. http://dx.doi.org/10.1016/j. diabet.2012.10.005.

[75] Richardson LC, Pollack LA. Therapy insight: Influence of type 2 diabetes on the development, treatment and outcomes of cancer. Nat Clin Pract Oncol 2005;2:48-53.

[76] Rinke A, Wittenberg M, Schade-Brittinger C, Aminossadati B, Ronicke E, Gress TM, et al. PROMID Study Group 2009 Placebo-controlled, double-blind, prospective, randomized study on the effect of octreotide LAR in the control of tumor growth in patients with metastatic neuroendocrine midgut tumors: a report from the PROMID Study Group. J Clin Oncol 2009;27:4656-63.

[77] Sah RP, Nagpal SJ, Mukhopadhyay D, Chari ST. New insights into pancreatic cancer-induced paraneoplastic diabetes. Nat Rev Gastroenterol Hepatol 2013;10:423-33. http://dx.doi.org/10.1038/nrgastro.2013.49.

[78] Santen RJ, Mannen A. Diagnosis and management of endocrine-related tumors. Springer Science \& Business Media; 2012.

[79] Singh G, Eng J, Raufman JP. Use of 125I-[Y39] exendin-4 to characterize exendin receptors on dispersed pancreatic acini and gastric chief cells from guinea pig. Regulatory Peptides 1994;53:47-59.

[80] Singh V, Brendel MD, Zacharias S, Mergler S, Jahr H, Wiedenmann B, et al. Characterization of somatostatin receptor subtype-specific regulation of insulin and glucagon secretion: an in vitro study on isolated human pancreatic islets. J Clin Endocrinol Metab 2007;92:673-80.

[81] Slezak LA, Andersen DK. Pancreatic resection: effects on glucose metabolism. World J Surg 2001;25:452-60.

[82] Soares HP, Ni Y, Kisfalvi K, Sinnett-Smith J, Rozengurt E. Different patterns of Akt and ERK feedback activation in response to rapamycin, active-site mTOR inhibitors and metformin in pancreatic cancer cells. PLoS ONE 2013:8:e57289.

[83] Theodoraki A, Khoo B, Hamda A, Grillo F, Meyer T, Bouloux PM. Malignant somatostatinoma presenting with diabetic ketoacidosis and inhibitory syndrome: pathophysiologic considerations. Endocrine Practice 2010;16:835-7.

[84] Umlauft M, Radojewski P, Spanjol PM, Dumont R, Marincek N, Kollar A, et al. Diabetes mellitus and its effects on all-cause mortality after radiopeptide therapy for neuroendocrine tumors. J Nucl Med 2017;58:97-102. http://dx.doi.org/10 2967/jnumed.116.180687.

[85] Vezzosi D, Bennet A, Rochaix P, Courbon F, Selves J, Pradere B, et al. Octreotide in insulinoma patients: efficacy on hypoglycemia, relationships with Octreoscan scintigraphy and immunostaining with anti-sst2A and anti-sst5 antibodies. Eur J Endocrinol 2005:152:757-67.

[86] Wermers RA, Fatourechi V, Wynne AG, Kvols LK, Lloyd RV. The glucagonoma syndrome. Clinical and pathologic features in 21 patients. Medicine (Baltimore) 1999;75:53-63.

[87] Wicki A, Wild D, Storch D, Seemayer C, Gotthardt M, Behe M, et al. [Lys40(AhxDTPA-111In)NH2]-Exendin-4 is a highly efficient radiotherapeutic for glucagonlike peptide-1 receptor-targeted therapy for insulinoma. Clin Cancer Res 2007;13:3696-705.

[88] Wolin EM, Jarzab B, Eriksson B, Walter T, Toumpanakis C, Morse MA, et al. Phase III study of pasireotide long-acting release in patients with metastatic neuroendocrine tumors and carcinoid symptoms refractory to available somatostatin analogues. Drug Des Dev Therapy 2015;9:5075-86. http://dx.doi.org/10.2147/DDDT. S84177.

[89] Yalcin S, Öberg K. Neuroendocrine Tumours - Diagnosis and Management. Berlin 
Heidelberg: Springer; 2015. http://dx.doi.org/10.1007/978-3-662-45215-8.

[90] Yao JC, Fazio N, Singh S, Buzzoni R, Carnaghi C, Wolin E, et al. Everolimus for the treatment of advanced, non-functional neuroendocrine tumours of the lung or gastrointestinal tract (RADIANT-4): a randomised, placebo-controlled, phase 3 study. Lancet 2016;387:968-77. http://dx.doi.org/10.1016/S0140-6736(15) 00817-X.

[91] Yao JC, Hassan M, Phan A, Dagohoy C, Leary C, Mares JE, et al. One hundred years after 'carcinoid': epidemiology of and prognostic factors for neuroendocrine tumors in 35,825 cases in the United States. J Clin Oncol 2008;26:3063-72.

[92] Yao JC, Lombard-Bohas C, Baudin E, Kvols LK, Rougier P, Ruszniewski P, et al. Daily oral everolimus activity in patients with metastatic pancreatic neuroendocrine tumors after failure of cytotoxic chemotherapy: a phase II trial. J Clin Oncol 2010;28:69-76. http://dx.doi.org/10.1200/JCO.2009.24.2669.

[93] Yao JC, Shah MH, Ito T. Everolimus for advanced pancreatic neuroendocrine tumors. N Engl J Med 2011;364:514-23.
[94] Yin M, Zhou J, Gorak EJ, Quddus F. Metformin is associated with survival benefit in cancer patients with concurrent type 2 diabetes: a systematic review and metaanalysis. Oncologist 2013;18:1248-55.

[95] Yoshida K, Imamura CK, Hara K, Mochizuki M, Tanigawara Y. Effect of everolimus on the glucose metabolic pathway in mouse skeletal muscle cells (C2C12). Metabolomics 2017;13:98. http://dx.doi.org/10.1007/s11306-017-1236-5.

[96] Zambre Y, Ling Z, Chen MC, Hou X, Woon CW, Culler M, et al. Inhibition of human pancreatic islet insulin release by receptor-selective somatostatin analogs directed to somatostatin receptor subtype 5. Biochem Pharmacol 1999;57:1159-64.

[97] Zhan HX, Cong L, Zhao YP, Zhang TP, Chen G. Risk factors for the occurrence of insulinoma: a case-control study. Hepatobiliary Pancreatic Dis Int 2013;12:324-8.

[98] Zhong J, Ali AN, Voloschin AD, Liu Y, Curran Jr WJ, Crocker IR, et al. Bevacizumabinduced hypertension is a predictive marker for improved outcomes in patients with recurrent glioblastoma treated with bevacizumab. Cancer 2015;121:1456-62. http://dx.doi.org/10.1002/cncr.29234. 